\title{
Risk factors of continuous renal replacement therapy following total aortic arch replacement under moderate hypothermia
}

\author{
Bo Li ${ }^{1 \#}$, Qing-Liang Chen ${ }^{1 \#}$, Bo-Chen Yao ${ }^{1}, \mathrm{Nan}_{\mathrm{Jiang}}{ }^{1}$, Feng Zhao ${ }^{1}, \mathrm{Min}_{\mathrm{Ren}}{ }^{2}$, Jing Sun ${ }^{1}$, Li-Na Xu ${ }^{1}$, \\ Zhi-Gang Guo ${ }^{1}$
}

${ }^{1}$ Department of Cardiovascular Surgery, Tianjin Chest Hospital, Tianjin, China; ${ }^{2}$ Tianjin Institute of Cardiovascular Diseases, Tianjin, China

Contributions: (I) Conception and design: All authors; (II) Administrative support: M Ren, N Jiang, F Zhao, LN Xu, ZG Guo; (III) Provision of study materials or patients: B Li, QL Chen, BC Yao; (IV) Collection and assembly of data: B Li, QL Chen, BC Yao; (V) Data analysis and interpretation: B Li, QL Chen, BC Yao, M Ren; (VI) Manuscript writing: All authors; (VII) Final approval of manuscript: All authors.

\#These authors contributed equally to this work.

Correspondence to: Qing-Liang Chen. Department of Cardiovascular Surgery, Tianjin Chest Hospital, 261 Taierzhuang Road, Tianjin 300001, China. Email: 314067280@qq.com; Zhi-Gang Guo. Tianjin Chest Hospital, 261 Taierzhuang Road, Tianjin 300001, China. Email: docboblee86@gmail.com.

Background: Stanford type A aortic dissection (TAAD) has a sudden onset and high mortality, and emergency total aortic arch replacement (TAAR) is the main treatment option for TAAD. The mortality rate of patients with postoperative acute kidney injury (AKI) combined with continuous renal replacement therapy (CRRT) is remarkable higher than that of patients without AKI. However, incidence of AKI and risk factors for CRRT following TAAR isn't entirely understood.

Methods: From October 2018 to March 2021, all patients with Stanford type A dissection who underwent total arch replacement surgery under MHCA were enrolled. According to whether CRRT treatment was performed, participants were divided into a CRRT group $(n=49)$ and control group $(n=72)$. Both groups incorporated the brain protection strategy of moderate hypothermia, and the left common carotid artery and the innominate artery were perfused anteriorly. Relevant medical data was collected.

Results: Age, gender, and a history of smoking and drinking were not significantly different between the 2 groups $(\mathrm{P}>0.1)$. There were statistical differences between the 2 groups in aortic sinus diameter and Bentall procedure $(\mathrm{P} \leq 0.05)$. Univariate analysis revealed that fresh frozen plasma was a protective factor $(\mathrm{P}<0.05)$ and the intraoperative transfusion volume of red blood cells, platelets, fresh frozen plasma, autologous blood used for intraoperative bleeding, aortic sinus diameter, and Bentall procedure were risk factors $(\mathrm{P}<0.1)$. Multivariate analysis showed that the Bentall procedure and intraoperative bleeding were risk factors for CRRT $(\mathrm{P}<0.05)$, and the aortic sinus diameter and intraoperative transfusion score were also risk factors for CRRT $(\mathrm{P}<0.05)$. Receiver operating characteristic $(\mathrm{ROC})$ analysis demonstrated that the model of aortic sinus diameter and intraoperative transfusion score had more significantly different discriminatory powers.

Conclusions: The Bentall procedure, intraoperative bleeding, aortic sinus diameter, and intraoperative transfusion score were risk factors for postoperative CRRT. The model of aortic sinus diameter and intraoperative transfusion score had more significantly different discriminatory powers.

Keywords: Acute kidney injury (AKI); continuous renal replacement therapy (CRRT); moderate hypothermia circulatory arrest (MHCA); type A aortic dissection (TAAD); total aortic arch replacement (TAAR)

Submitted Jul 19, 2021. Accepted for publication Aug 30, 2021.

doi: 10.21037/atm-21-3905

View this article at: https://dx.doi.org/10.21037/atm-21-3905 


\section{Introduction}

Stanford type A aortic dissection (TAAD) is characterized by a sudden onset and high mortality (1). Currently, total aortic arch replacement (TAAR) is routinely performed using deep hypothermic circulatory arrest (DHCA) for the treatment of TAAD (2). However, the operative longterm use of DHCA damages multiple organs. Acute kidney injury (AKI), occurs in approximately $40-50 \%$ of patients after aortic surgery using DHCA (3). Approximately 6\% of patients with $\mathrm{AKI}$ require continuous renal replacement therapy (CRRT).

In recent years, surgical treatment of type A aortic dissection (TAAD) has been performed using moderate hypothermia hypothermic circulatory arrest (MHCA) in many medical centers to reduce the incidence of postoperative complications $(4,5)$. However, AKI still occasionally occurs, and the incidence of AKI and risk factors for CRRT following TAAR under moderate hypothermia have rarely been reported. This singlecenter study retrospectively analyzed 121 patients with TAAD undergoing TAAR using MHCA and discusses the incidence of AKI and risk factors for CRRT following TAAD, with an aim to protect postoperative renal function and improve clinical outcomes. We present the following article in accordance with the STROBE reporting checklist (available at https://dx.doi.org/10.21037/atm-21-3905).

\section{Methods}

\section{General data}

All patients with TAAD who underwent TAAR using MHCA at this center from October 2018 to March 2021 were considered potential participants. Among them, those with acute aortic dissection undergoing surgical treatment $3 \mathrm{~d}$ after the onset were included in this study. Patients were excluded based on the exclusion criteria, as follows: (I) TAAD involved the renal artery, or a history of acute or chronic renal insufficiency with/without elevated creatinine levels; (II) preoperative pericardial tamponade affecting hemodynamic stability and requiring maintenance of circulation by vasoactive agents; (III) TAAD involved the iliac artery, resulting in cool skin temperature of the lower extremities, a weakened pulse, significantly elevated myoglobin levels, and postoperative ischemia-reperfusion injury. Finally, a total of 121 patients were included. All participants underwent computed tomography angiography (CTA) of the aorta and echocardiography to identify the extent of diagnosis and lesion and the involvement of the coronary artery (Table 1). The study was approved by Institutional Ethics Review Board of Tianjin Chest Hospital (2018-2123), and individual consent for this retrospective analysis was waived. All procedures performed in this study involving human participants were in accordance with the Declaration of Helsinki (as revised in 2013).

\section{Study methods}

\section{Criteria of CRRT therapy}

The criteria for initiation of CRRT were as follows: (I) patients were admitted to the intensive care unit due to more than $6 \mathrm{~h}$ of continuous anuria, and the urine volume was $<200 \mathrm{~mL}$ for over $10 \mathrm{~h}$; (II) hyperkalemia, when serum potassium concentration $>6 \mathrm{mmol} / \mathrm{L}$, marked improvements were not observed in patients routinely treated with diuretics; (III) severe metabolic acidosis ( $\mathrm{pH}<7.2$ ) could also not be improved, and patients showed hemodynamic instability; (IV) serum creatinine (sCr) $\geq 300 \mu \mathrm{mol} / \mathrm{L}$; (V) volume overload, with or without pulmonary edema; and poor response to conventional diuretics.

\section{Methods for the implementation of CRRT}

A double-channel catheter was placed through percutaneous jugular puncture to establish vascular access. The hemodialysis machine (Prismaflex, Baxter Healthare Corp., Deerfield, IL, USA) and M100 filter (Prismaflex) were used in our center. Most participants were treated using continuous veno-venous hemofiltration (CVVH) mode and we changed the mode according to the different participant conditions. Modified port composition was used in CRRT therapy and pre-dilution and post-dilution at a ratio of 50:50 were adopted. The flow rate of the substitution fluid was 2-4 L/h. Low-molecular-weight heparin was used for anticoagulation, and heparin-free CRRT was administered to participants with coagulopathy.

\section{Surgical method}

Participants were placed in a supine position, and they received intravenous and inhalational anesthesia. The procedure was performed through a full median sternotomy and cardiopulmonary bypass (CPB) was established via femoral artery and superior and inferior vena cava cannulation. A left atrial catheter was implanted. The MHCA was performed at $32{ }^{\circ} \mathrm{C}$ and the ascending aorta was opened. The thrombus was cleared, the proximal false 
Table 1 Preoperative variables

\begin{tabular}{|c|c|c|c|c|}
\hline Variable & Control group & CRRT group & T-value or $\chi^{2}$ value & $P$ value \\
\hline Age (year) & $57.2 \pm 10.2$ & $56.6 \pm 12.6$ & -0.27 & 0.79 \\
\hline Smoking, n (\%) & $38(52.8)$ & $27(55.1)$ & 0.06 & 0.80 \\
\hline Drinking, n (\%) & $22(30.6)$ & $12(24.5)$ & 0.53 & 0.47 \\
\hline Hypertension & $55(76.4)$ & $39(79.6)$ & 0.17 & 0.68 \\
\hline Diabetes & $2(2.8)$ & $0(0.0)$ & 1.38 & 0.24 \\
\hline Cerebrovascular accident & $4(0.0)$ & $2(2.8)$ & 0.13 & 0.71 \\
\hline Coronary heart disease & $8(16.3)$ & $8(11.1)$ & 0.69 & 0.41 \\
\hline Ascending aorta diameter (mm) & $47.2 \pm 7.1$ & $46.9 \pm 6.6$ & -0.20 & 0.84 \\
\hline LAEDD (mm) & $36.9 \pm 4.4$ & $36.1 \pm 6.1$ & -0.76 & 0.45 \\
\hline LVEDD (mm) & $52.6 \pm 6.2$ & $52.4 \pm 7.8$ & -0.15 & 0.88 \\
\hline $\operatorname{LVEF}(\mathrm{mm})$ & $56.0 \pm 5.0$ & $54.9 \pm 7.5$ & -0.85 & 0.40 \\
\hline Mitral valvular lesion, n (\%) & $8(11.1)$ & $3(6.1)$ & 0.88 & 0.35 \\
\hline Aortic valvular lesions, n (\%) & $27(37.5)$ & $25(51.0)$ & 2.18 & 0.14 \\
\hline \multicolumn{5}{|l|}{ Combined surgery, n (\%) } \\
\hline Ascending aorta replacement & $39(54.2)$ & $22(44.9)$ & 0.31 & 0.58 \\
\hline
\end{tabular}

LAEDD, left atrial end-diastolic dimension; LVEDD, left ventricular end-diastolic dimension; LVEF, left ventricular ejection fraction; CRRT, continuous renal replacement therapy; AVR/AVP, aortic valve replacement/aortic valve repair; CABG, coronary artery bypass graft; MVR, mitral valve replacement; CRRT, continuous renal replacement therapy.

lumen was eliminated by continuous mattress suture, an artificial blood vessel was implanted, circulatory arrest was confirmed at a rectal temperature of $28{ }^{\circ} \mathrm{C}$, and antegrade selective cerebral perfusion was performed. An open stented graft was deployed into the descending aorta. Subsequently, distal anastomosis was performed between the proximal end of the stent graft and a 4-branched graft. Distal reperfusion began following the completion of proximal anastomosis. The left carotid artery was reconstructed first, then the left subclavian artery, and finally, the innominate artery. Operative variables were shown in Table 2. The simultaneous conduction of other procedures depended on the damage of the aortic root (Table 1).

\section{Statistical analysis}

The software SPSS 21.0 (IBM Corp., Armonk, NY, USA) was used for statistical analyses. Continuous, normally distributed variables were expressed as mean \pm standard deviation (SD), and were compared using Student's $t$-test. Categorical variables were compared using Pearson's $\chi^{2}$ test or Fisher's exact test as appropriate. Variables with a 
Table 2 Operative variables

\begin{tabular}{|c|c|c|c|c|}
\hline Variable & Control group & CRRT group & T-value or $\chi^{2}$ value & $P$ value \\
\hline CPB time (min) & $177.0 \pm 42.4$ & $191.4 \pm 53.6$ & 1.64 & 0.10 \\
\hline Aortic cross-clamp time (min) & $102.4 \pm 27.6$ & $107.8 \pm 30.9$ & 1.00 & 0.32 \\
\hline Circulatory arrest time (min) & $15.1 \pm 8.7$ & $13.1 \pm 11.6$ & -1.04 & 0.30 \\
\hline Circulatory arrest temperature (anal temperature ${ }^{\circ} \mathrm{C}$ ) & $27.9 \pm 2.3$ & $27.6 \pm 1.8$ & -0.71 & 0.48 \\
\hline \multicolumn{5}{|l|}{ Intraoperative blood product } \\
\hline Red blood cells $(U)$ & $1.1 \pm 2.3$ & $2.4 \pm 3.4$ & 2.41 & 0.02 \\
\hline Platelets (therapeutic dose) & $1.1 \pm 0.7$ & $1.7 \pm 0.8$ & 3.86 & 0.00 \\
\hline \multicolumn{5}{|l|}{ Drainage volume } \\
\hline Intraoperative bleeding (mL) & $1,270.0 \pm 508.4$ & $1,577.7 \pm 824.6$ & 2.47 & 0.02 \\
\hline Postoperative drainage $(\mathrm{mL})$ & $1,036.6 \pm 801.4$ & $1,185.2 \pm 923.3$ & 0.91 & 0.37 \\
\hline
\end{tabular}

CPB, cardiopulmonary bypass; CRRT, continuous renal replacement therapy.

$\mathrm{P}<0.1$ in univariate analysis were assessed with multivariate analysis using a forward stepwise binary logistic regression model, and a two-sided $\mathrm{P}$ value $<0.1$ was considered statistically significant. Every multivariable model was tested for reliability with the Hosmer-Lemeshow statistic. The scoring indicators were used as inspection variables, and the CRRT treatment was used as the state variable for plotting receiver operator characteristic (ROC) curve. The diagnostic cut-off values were determined using the Youden index. Finally, the discriminatory ability of every logistic regression model developed was tested using the area under ROC curve ( $\left.\mathrm{AUC}_{\mathrm{ROC}}\right)$.

\section{Results}

\section{Analysis of general information}

In this study, participants were divided into the CRRT $(\mathrm{n}=49$; average age: $56.62 \pm 12.6$; male: $35,71.4 \%)$ and control ( $\mathrm{n}=72 ; 57.2 \pm 10.2$; male: $48,66.7 \%)$ groups depending on whether they accepted CRRT treatment after surgery. Age, gender, and a history of smoking and drinking were not significantly different between the 2 groups $(\mathrm{P}>0.1)$. However, the comparison of complications, echocardiography, and concomitant cardiac procedures detected statistical differences between the 2 groups in aortic sinus diameter and Bentall procedure $(\mathrm{P} \leq 0.05)$.

\section{Trend of changes in postoperative sCr levels}

On day 1 after surgery, sCr in the CRRT group increased remarkably, with an average value of $214.04 \pm 79.51 \mu \mathrm{mol} / \mathrm{L}$, whereas that in the control group was $127.32 \pm 58.08 \mu \mathrm{mol} / \mathrm{L}$. In the CRRT group, despite the conduction of CRRT treatment, the trend of changes in sCr levels was not obvious on days 2-4 after surgery, and the average value of sCr was $264.20 \pm 111.76 \mu \mathrm{mol} / \mathrm{L}$ on day 4 after surgery. In the control group, the sCr levels decreased noticeably within 4 days after surgery, and the average value of sCr was $104.24 \pm 76.00 \mu \mathrm{mol} / \mathrm{L}$ on day 4 after surgery (Figure 1).

\section{Single risk factor analysis of perioperative and intraoperative variables}

Comparison of perioperative variables between the 2 groups indicated statistical differences in the intraoperative transfusion volume of red blood cells, platelets, fresh frozen plasma, autologous blood used for intraoperative bleeding, aortic sinus diameter, and Bentall procedure $(\mathrm{P}<0.1)$. Univariate analysis found that fresh frozen plasma was a 
protective factor $(\mathrm{P}<0.05)$ and the other variables above were risk factors $(\mathrm{P}<0.1)$ (Table 3).

\section{Formulation of intraoperative transfusion score}

As the red blood cells, platelets, fresh frozen plasma, and autologous blood used for intraoperative transfusion belonged to the same class of indicators in this study, we formulated intraoperative transfusion scores using cutoff values which were determined by ROC curve. For protective factors, the number "1"/" 0 " was assigned if transfusion volumes were greater than/less than or equal to the cutoff value. For risk factors, the number "0"/"1"

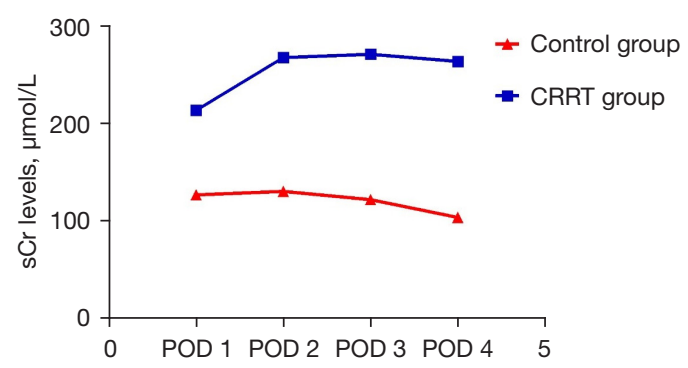

Figure 1 Changes in postoperative sCr levels. CRRT, continuous renal replacement therapy; $\mathrm{sCr}$, serum creatinine. was assigned if transfusion volumes were greater than/less than or equal to the cutoff value (Table 4). The scores in each participant were analyzed, and a univariate analysis was performed. A significant difference between 2 groups was detected in intraoperative transfusion score $(\mathrm{P}<0.05)$ (Table 3).

\section{Multivariate analysis for CRRT}

Correlation analysis revealed a significant correlation between intraoperative bleeding and intraoperative transfusion score $(\mathrm{P}<0.05)$. Therefore, multivariate analysis was respectively conducted for the aforementioned two indexes. Table 5 shows that the Bentall procedure and intraoperative bleeding were risk factors for CRRT $(\mathrm{P}<0.05)$, and Table 6 shows that the aortic sinus diameter and intraoperative transfusion score were risk factors for CRRT $(\mathrm{P}<0.1)$.

\section{Comparison of precision between 2 models}

Analysis of the ROC showed that the 2 models had significantly different discriminatory powers. The results demonstrated a significant improvement in AUC in the model where aortic sinus diameter and intraoperative transfusion score were included (Table 7 and Figure 2).

Table 3 Univariate analysis of variables associated with CRRT

\begin{tabular}{lccccc}
\hline Risk factors & B-value & Wald $\chi^{2}$ value & P value & OR value & $95 \% \mathrm{Cl}$ \\
\hline Aortic sinus diameter $(\mathrm{mm})$ & 0.066 & 3.729 & 0.053 & 1.068 & $0.999-1.141$ \\
Bentall procedure $[\mathrm{n}(\%)]$ & 1.066 & 6.048 & 0.014 & 2.903 & $1.242-6.788$ \\
Red blood cells $(\mathrm{U})$ & 0.159 & 5.120 & 0.024 & 0.001 & 2.826 \\
Platelets (therapeutic dose) & 1.039 & 12.033 & 0.010 & 0.999 & $0.998-1.000$ \\
Fresh frozen plasma $(\mathrm{mL})$ & -0.001 & 6.685 & 0.004 & 1.001 & $1.000-1.002$ \\
Autologous blood $(\mathrm{mL})$ & 0.001 & 8.391 & 0.022 & 1.001 & $1.000-1.001$ \\
Intraoperative bleeding $(\mathrm{mL})$ & 0.001 & 5.228 & 0.000 & 3.452 & $2.075-5.744$ \\
Intraoperative transfusion score & 1.239 & 22.744 &
\end{tabular}

CRRT, continuous renal replacement therapy; OR, odds ratio; $\mathrm{Cl}$, confidence interval.

Table 4 Intraoperative transfusion score standard

\begin{tabular}{lcccc}
\hline Assignment & Red blood cells $(\mathrm{U})$ & Platelets (therapeutic dose) & Fresh frozen plasma $(\mathrm{mL})$ & Autologous blood $(\mathrm{mL})$ \\
\hline 1 & $>1.5$ & $>1.5$ & $\leq 741.5$ & $>1,375$ \\
0 & $\leq 1.5$ & $\leq 1.5$ & $>741.5$ & $\leq 1,375$ \\
\hline
\end{tabular}


Table 5 Multivariate analysis for CRRT [1]

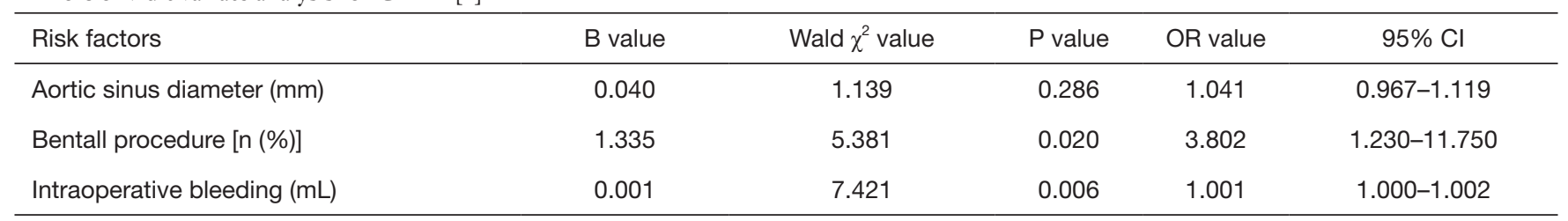

Hosmer-Lemeshow test $\chi^{2}=5.592, \mathrm{P}=0.693$. CRRT, continuous renal replacement therapy; OR, odds ratio; $\mathrm{Cl}$, confidence interval.

Table 6 Multivariate analysis for CRRT [2]

\begin{tabular}{lccccc}
\hline Risk factors & B value & Wald $\chi^{2}$ value & P value & OR value & 95\% Cl \\
\hline Aortic sinus diameter $(\mathrm{mm})$ & 0.082 & 3.447 & 0.063 & 1.086 & $0.995-1.185$ \\
Bentall procedure $[\mathrm{n}(\%)]$ & 0.881 & 1.983 & 0.159 & 2.413 & $0.708-8.221$ \\
Intraoperative transfusion score & 1.345 & 18.090 & 0.001 & 3.839 & $2.065-7.137$
\end{tabular}

Hosmer-Lemeshow test $\chi^{2}=12.741, \mathrm{P}=0.121$. CRRT, continuous renal replacement therapy; OR, odds ratio; Cl, confidence interval.

Table 7 Analysis of the predictive abilities of the 2 models

\begin{tabular}{llll}
\hline Model indexes & $\mathrm{ROC}$ & $\mathrm{P}$ value & $95 \% \mathrm{Cl}$ \\
\hline Bentall procedure and intraoperative bleeding & 0.756 & 0.000 & $0.650-0.862$ \\
Aortic sinus diameter and Intraoperative transfusion score & 0.837 & 0.000 & $0.754-0.920$ \\
\hline
\end{tabular}

$\mathrm{ROC}$, receiver operating characteristic; $\mathrm{Cl}$, confidence interval.

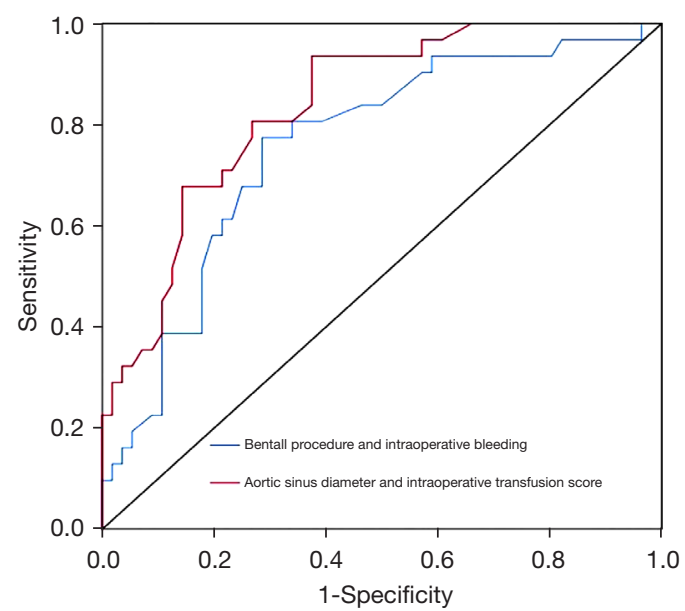

Figure 2 Analysis of the ROC curves for the two models. ROC, receiver operating characteristic.

\section{Discussion}

The condition of TAAD has a sudden onset and high mortality (6), and emergency TAAR is the main treatment option for TAAD. Over the years, there has been an increase in the success rate of emergency TAAR and decrease in postoperative mortality, attributable to the fact that MHCA can effectively shorten the duration of cooling and rewarming and reduce postoperative ischemiareperfusion injury to organs; however, it cannot prevent postoperative complications. The mortality rate (15-30\%) of patients with postoperative AKI is 10-20 times that of patients without AKI. Therefore, AKI is an independent risk factor contributing to postoperative death (7). Patients with AKI, especially those with AKI after TAAR procedure, may experience water-sodium retention, azotemia, internal environment disorder, and fluid overload, and should promptly undergo CRRT (8).

In this study, we selected patients undergoing TAAR in this center, and factors that could lead to AKI and those associated with postoperative CRRT were excluded. The potential impact of perioperative indexes on postoperative CRRT was analyzed. After grouping based on whether CRRT was performed, the sCr level in the CRRT group was remarkably higher than that in the control group within 4 days after surgery. To date, the Kidney Disease: Improving Global Outcomes (KDIGO) guideline has 
been extensively used to determine the optimum time to implement CRRT (9). AKI is defined as: increase in sCr by $\geq 0.3 \mathrm{mg} / \mathrm{dL}$ within 48 hours, increase in $\mathrm{sCr}$ to $\geq 1.5$ times baseline, which is known or presumed to have occurred within the prior 7 days or urine volume $<0.5 \mathrm{~mL} / \mathrm{kg} / \mathrm{h}$ for 6 hours. Recently, an increasing number of studies have suggested that earlier implementation of CRRT could lead to better clinical efficacy in treating AKI (10-13). Given the special characteristics of cardiac surgery, there are high requirements for the control of fluid load volume and internal environment within $24 \mathrm{~h}$ after surgery, and the time to implement CRRT cannot be determined in accordance with the KDIGO guidelines. In this study, the criteria for CRRT in this center were established based on specific conditions, and compared with the criteria for CRRT used in other cardiac centers, there was no marked difference.

This study revealed that preoperative aortic sinus diameter, combined Bentall procedure, intraoperative blood products, and intraoperative bleeding were risk factors for postoperative CRRT. The aortic sinus diameter was positively correlated with postoperative CRRT, which is believed to be because a greater aortic sinus diameter leads to greater difficulty in vascular anastomosis and a longer surgical duration, thereby causing an increase in the frequency of performing CRRT. A longer surgical duration leads to aggravated destruction of blood constituents and ischemia-reperfusion injury to various organs. Additionally, factors such as surgical trauma can induce systemic inflammatory response syndrome (SIRS) (14) and activate inflammatory factors that induce damage to the vascular endothelium cells upon release, which thereby damages organs and tissues and the renal filtration system when reperfusion occurs. To date, multiple studies have validated that prolonged $\mathrm{CPB}$ is also a risk factor. Especially after total aortic arch replacement with DHCA, prolonged $\mathrm{CPB}$ and aortic occlusion time were risk factors for acute kidney injury. In this study, CPB duration was not included as a risk factor. This may be attributed to the shortened $\mathrm{CPB}$ duration due to MHCA used in TAAR procedure, and as a result, a long CPB duration can be effectively avoided. The Bentall procedure is positively correlated with postoperative CRRT, which primarily involves complicated surgical procedures and prolonged surgical duration.

Intraoperative bleeding and transfusion of blood products are risk factors for postoperative CRRT. Given the difficulty of performing TAAR and long surgical duration, increase in the amount of intraoperative bleeding indicates that more units of banked blood would be transfused during surgery. Therefore, the amount of intraoperative bleeding is closely associated with the quantities of intraoperative blood products transfused. The study findings showed that intraoperative transfusion of red blood cells, platelets, and autologous blood could lead to an increase in the frequency of performing postoperative CRRT, which is consistent with the reports of previous studies $(15,16)$. Regarding the transfusion of a large number of banked red blood cells, platelets, and autologous blood in the surgery, red blood cell fragments, and microthrombus can block the renal tubules, thereby inducing AKI (17). Hemoglobin release by fragmented red blood cells is an important factor contributing to occlusion of the renal tubules. A longer storage of red blood cells leads to reduction in the oxygen delivery capacity and internal milieu regulation capability, leading to the release of cytotoxins, vasoconstriction, and oxidative stress. The aforementioned factors can aggravate AKI $(18,19)$, and their impact is positively correlated with transfusion volume. Intraoperative bleeding also can aggravate intraoperative hemodynamic instability, which can further reduce renal perfusion (20) and induce AKI. In addition, the study findings suggested that the intraoperative use of plasma was negatively correlated with postoperative CRRT, indicating that the use of fresh frozen plasma was a protective factor against AKI. This may be because fresh frozen plasma can effectively maintain stable circulation, and coagulation factors in fresh frozen plasma can strengthen the coagulation function of patients and reduce the consumption of other blood products (21). The specific mechanisms remain to be validated.

The most unique part in my paper is that we formulated intraoperative transfusion scores using cutoff values which were determined by ROC curve. Because we find intraoperative transfusion volume of blood product has a strong relationship with CRRT. But the red blood cells, platelets, fresh frozen plasma, and autologous blood for intraoperative transfusion belong to the same class of indicators in this study. So, this intraoperative transfusion scores can solve the problem that same class data can't compare with each other. This kind statistic method is not common in other articles. This study had some limitations. It was a single-center trial, surgeon skills and experience, and improvement in surgical procedures significantly affected prognoses. Additionally, the small sample and type II errors inevitably may have led to biased results. Therefore, a future retrospective study with a larger sample size should be performed to reduce such bias. In summary, TAAR takes time and involves complicated surgical 
procedures. Surgical techniques should be improved in order to reduce intraoperative bleeding and cause minimal damage to blood-formed elements, and indications for intraoperative transfusion should be strictly followed. The surgical duration and CPB time should be maximally shortened, which may be protective against AKI.

\section{Acknowledgments}

Funding: This work was supported by Major Projects of Disease Prevention and Cure of Tianjin Science and Technology Commission "Clinical diagnosis and treatment research of elderly patients with aortic dissecting aneurysm in Tianjin" (18ZXDBSY00160).

\section{Footnote}

Reporting Checklist: The authors have completed the STROBE reporting checklist. Available at https://dx.doi. org/10.21037/atm-21-3905

Data Sharing Statement: Available at https://dx.doi. org/10.21037/atm-21-3905

Conflicts of Interest: All authors have completed the ICMJE uniform disclosure form (available at https://dx.doi. org/10.21037/atm-21-3905). The authors report that this work was supported by Major Projects of Disease Prevention and Cure of Tianjin Science and Technology Commission (18ZXDBSY00160), payments to Tianjin Chest Hospital. The authors have no other conflicts of interest to declare.

Ethical Statement: The authors are accountable for all aspects of the work in ensuring that questions related to the accuracy or integrity of any part of the work are appropriately investigated and resolved. The study was approved by Institutional Ethics Review Board of Tianjin Chest Hospital (2018-2123), and individual consent for this retrospective analysis was waived. All procedures performed in this study involving human participants were in accordance with the Declaration of Helsinki (as revised in 2013).

Open Access Statement: This is an Open Access article distributed in accordance with the Creative Commons Attribution-NonCommercial-NoDerivs 4.0 International License (CC BY-NC-ND 4.0), which permits the non- commercial replication and distribution of the article with the strict proviso that no changes or edits are made and the original work is properly cited (including links to both the formal publication through the relevant DOI and the license). See: https://creativecommons.org/licenses/by-nc-nd/4.0/.

\section{References}

1. Bressler M, Klein E, Shayowitz M, et al. A Case of Late Diagnosis of Acute Aortic Dissection Involving the Carotid Arteries in a Patient Presenting with Signs of Acute Ischemic Stroke. Am J Case Rep 2020;21:e919271.

2. Fang Z, Wang G, Liu Q, et al. Moderate and deep hypothermic circulatory arrest has a comparable effect on acute kidney injury after total arch replacement with frozen elephant trunk procedure in type A aortic dissection. Interact Cardiovasc Thorac Surg 2019;29:130-6.

3. Li F, Liu N, Dong P, et al. Analysis of factors related to acute renal failure post deep hypothermia circulatory arrest surgery of type A aorta dissection surgery. Zhonghua Wai Ke Za Zhi 2013;51:1094-8.

4. El-Sayed Ahmad A, Risteski P, Ay M, et al. Moderate Hypothermic Circulatory Arrest $\left(\geq 28^{\circ} \mathrm{C}\right)$ with Selective Antegrade Cerebral Perfusion for Total Arch Replacement with Frozen Elephant Trunk Technique. Thorac Cardiovasc Surg 2019;67:345-50.

5. Yang P, Wu P, Liu X, et al. MiR-26b Suppresses the Development of Stanford Type A Aortic Dissection by Regulating HMGA2 and TGF- $\beta /$ Smad3 Signaling Pathway. Ann Thorac Cardiovasc Surg 2020;26:140-50.

6. Nakamura T, Mikamo A, Matsuno Y, et al. Impact of acute kidney injury on prognosis of chronic kidney disease after aortic arch surgery. Interact Cardiovasc Thorac Surg 2020;30:273-9.

7. Chien TM, Wen H, Huang JW, et al. Significance of preoperative acute kidney injury in patients with acute type A aortic dissection. J Formos Med Assoc 2019;118:815-20.

8. Sueyoshi K, Watanabe Y, Inoue T, et al. Predictors of long-term prognosis in acute kidney injury survivors who require continuous renal replacement therapy after cardiovascular surgery. PLoS One 2019;14:e0211429.

9. Khwaja A. KDIGO clinical practice guidelines for acute kidney injury. Nephron Clin Pract 2012;120:c179-84.

10. Rhee H, Jang GS, Han M, et al. The role of the specialized team in the operation of continuous renal replacement therapy: a single-center experience. BMC Nephrol 2017;18:332. 
11. Yoon BR, Leem AY, Park MS, et al. Optimal timing of initiating continuous renal replacement therapy in septic shock patients with acute kidney injury. Sci Rep 2019;9:11981.

12. Chen WY, Cai LH, Zhang ZH, et al. The timing of continuous renal replacement therapy initiation in sepsisassociated acute kidney injury in the intensive care unit: the CRTSAKI Study (Continuous RRT Timing in Sepsisassociated AKI in ICU): study protocol for a multicentre, randomised controlled trial. BMJ Open 2021;11:e040718.

13. Nadim MK, Forni LG, Mehta RL, et al. COVID-19associated acute kidney injury: consensus report of the 25th Acute Disease Quality Initiative (ADQI) Workgroup. Nat Rev Nephrol 2020;16:747-64.

14. Rangel-Frausto MS, Pittet D, Costigan M, et al. The natural history of the systemic inflammatory response syndrome (SIRS). A prospective study. JAMA 1995;273:117-23.

15. Tong J, Cao L, Liu L, et al. Impact of autologous platelet rich plasma use on postoperative acute kidney injury in type A acute aortic dissection repair: a retrospective cohort analysis. J Cardiothorac Surg 2021;16:9.

16. From the American Association of Neurological Surgeons (AANS), American Society of Neuroradiology (ASNR), Cardiovascular and Interventional Radiology Society of Europe (CIRSE), Canadian Interventional Radiology

Cite this article as: Li B, Chen QL, Yao BC, Jiang N, Zhao F, Ren M, Sun J, Xu LN, Guo ZG. Risk factors of continuous renal replacement therapy following total aortic arch replacement under moderate hypothermia. Ann Transl Med 2021;9(18):1448. doi: 10.21037/atm-21-3905
Association (CIRA), Congress of Neurological Surgeons (CNS), European Society of Minimally Invasive Neurological Therapy (ESMINT), European Society of Neuroradiology (ESNR), European Stroke Organization (ESO), Society for Cardiovascular Angiography and Interventions (SCAI), Society of Interventional Radiology (SIR), Society of NeuroInterventional Surgery (SNIS), and World Stroke Organization (WSO); Sacks D, Baxter B, et al. Multisociety Consensus Quality Improvement Revised Consensus Statement for Endovascular Therapy of Acute Ischemic Stroke. Int J Stroke 2018;13:612-32.

17. Yoshida M, Honma S. Regeneration of injured renal tubules. J Pharmacol Sci 2014;124:117-22.

18. Nakazawa D, Marschner JA, Platen L, et al. Extracellular traps in kidney disease. Kidney Int 2018;94:1087-98.

19. Faucon AL, Bobrie G, Clément O. Nephrotoxicity of iodinated contrast media: From pathophysiology to prevention strategies. Eur J Radiol 2019;116:231-41.

20. Beck J, Loron G, Masson C, et al. Monitoring Cerebral and Renal Oxygenation Status during Neonatal Digestive Surgeries Using Near Infrared Spectroscopy. Front Pediatr 2017;5:140.

21. Mathew J, Sankar P, Varacallo M. Physiology, Blood Plasma. 2021 Apr 28. In: StatPearls [Internet]. Treasure Island (FL): StatPearls Publishing; 2021 Jan. 